\title{
Regulatory network reconstruction reveals genes with prognostic value for chronic lymphocytic leukemia
}

\author{
Sally Yepes ${ }^{1 *}$, Maria Mercedes Torres ${ }^{1}$ and Liliana López-Kleine ${ }^{2}$
}

\begin{abstract}
Background: The clinical course of chronic lymphocytic leukemia (CLL) is highly variable; some patients follow an indolent course, but others progress to a more advanced stage. The mutational status of rearranged immunoglobulin heavy chain variable (IGVH) genes in CLL is a feature that is widely recognized for dividing patients into groups that are related to their prognoses. However, the regulatory programs associated with the IGVH statuses are poorly understood, and markers that can precisely predict survival outcomes have yet to be identified.

Methods: In this study, (i) we reconstructed gene regulatory networks in CLL by applying an information-theoretic approach to the expression profiles of 5 cohorts. (ii) We applied master regulator analysis (MRA) to these networks to identify transcription factors (TFs) that regulate an IGVH mutational status signature. The IGVH mutational status signature was developed by searching for differentially expressed genes between the IGVH mutational statuses in numerous CLL cohorts. (iii) To evaluate the biological implication of the inferred regulators, prognostic values were determined using time to treatment (TTT) and overall survival (OS) in two different cohorts.

Results: A robust IGVH expression signature was obtained, and various TFs emerged as regulators of the signature in most of the reconstructed networks. The TF targets expression profiles exhibited significant differences with respect to survival, which allowed the definition of a reduced profile with a high value for OS. TCF7 and its targets stood out for their roles in progression.
\end{abstract}

Conclusion: TFs and their targets, which were obtained merely from inferred regulatory associations, have prognostic implications and reflect a regulatory context for prognosis.

Keywords: CLL, IGVH mutational status, Regulatory networks

\section{Background}

Chronic lymphocytic leukemia (CLL) is a heterogeneous disease with variable clinical manifestations and evolution [1]. Two major molecular subtypes are recognized, which are characterized by a high or low number of somatic hypermutations in the variable region of the immunoglobulin genes. This feature is known as the immunoglobulin heavy chain variable (IGVH) gene mutational status and is related to prognostic evolution, in which patients with an unmutated IGVH status have a less favorable prognosis than patients with a mutated

\footnotetext{
* Correspondence: sl.yepes233@uniandes.edu.co

${ }^{1}$ Facultad de Ciencias, Departamento de Ciencias Biológicas, Universidad de los Andes, Bogotá D.C., Colombia

Full list of author information is available at the end of the article
}

IGHV gene [2, 3]. Other molecular biomarkers of progression in this disease include diverse cytogenetic rearrangements, gene mutations, and ZAP-70 expression [4-6]; however, these events do not appear to be fundamental agents in the leukemia process. Due to the importance of the IGVH status in disease course determination, several expression studies have focused on comparisons of the mutated IGVH vs. unmutated IGVH CLL forms [7-9]. However, these studies have identified genes that are not functionally related and therefore cannot elucidate biological mechanisms to distinguish between risk classes. Therefore, searching for the relevant prognostic biomarker surrogates for IGVH mutational status remains a necessity.

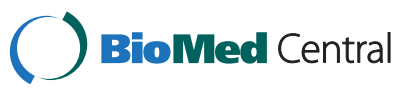

(c) 2015 Yepes et al. Open Access This article is distributed under the terms of the Creative Commons Attribution 4.0 International License (http://creativecommons.org/licenses/by/4.0/), which permits unrestricted use, distribution, and reproduction in any medium, provided you give appropriate credit to the original author(s) and the source, provide a link to the Creative Commons license, and indicate if changes were made. The Creative Commons Public Domain Dedication waiver (http://creativecommons.org/publicdomain/zero/1.0/) applies to the data made available in this article, unless otherwise stated. 
Several methods have been developed to identify expression signatures associated with prognosis. However, it is worth noting that markers are unstable and study dependent, often exhibiting poor overlap among studies and low classification power. According to Bae et al. [10], it is possible that expression signatures commonly contain cancer drivers and passengers, of which the latter are not directly involved in cancer progression. Therefore, it is of interest to search for regulators, such as transcription factors (TFs), that are causally responsible for the implementation of differential expression patterns and to evaluate their relation with progression and clinical outcome [11-13].

Here, in search of prognostic markers, we applied the ARACNE algorithm to find TFs that were involved in the differentiation process between IGVH subtypes. This algorithm is based on an information-theoretic approach that predicts potential functional associations among genes by identifying the statistical dependencies between their products [14]. ARACNE has been successfully applied in the search for master regulators and the study of clinical outcomes in different cancer models, the results of which can be subsequently validated through functional experiments. Some applications include the identification of c-MYC and BCL6 as critical genes in B-cell tumors $[15,16]$, master regulators with poor prognosis in breast cancer [11], cancer risk and master regulators for FGFR2 signaling [17], neuroblastoma and tumor progression [18] and multiple myeloma and its prognosis [19].
In this work, we reconstructed CLL regulatory networks using the ARACNE algorithm and used master regulator analysis (MRA) to identify candidate transcription factors that regulate an IGVH mutational status signature. The IGVH signature was developed through the identification of differentially expressed genes in a large number of samples using microarray meta-analysis. The principal intention here was to evaluate the implications of the inferred regulators and their targets for patient survival. Therefore, the candidate expression profiles were used to evaluate prognosis utilizing two measures of progression, time to treatment (TTT) and overall survival (OS).

The genes with prognostic implication identified in this work may represent reliable markers to predict outcomes because i) they were obtained through a method (MRA, which tested the significant intersections between the regulons that were represented in the ARACNE networks and the signature genes) that used as query a known prognostic marker in CLL, the IGVH mutational status, and ii) the genes that were inferred as key regulators exhibited significance using a Cox proportional hazards model with outcome indicators. Given the workflow, the identified genes reflect a regulatory context for prognosis rather than only differentially expressed genes.

\section{Methods}

A schematic description of the network reconstruction and regulator inference is represented in Fig. 1.

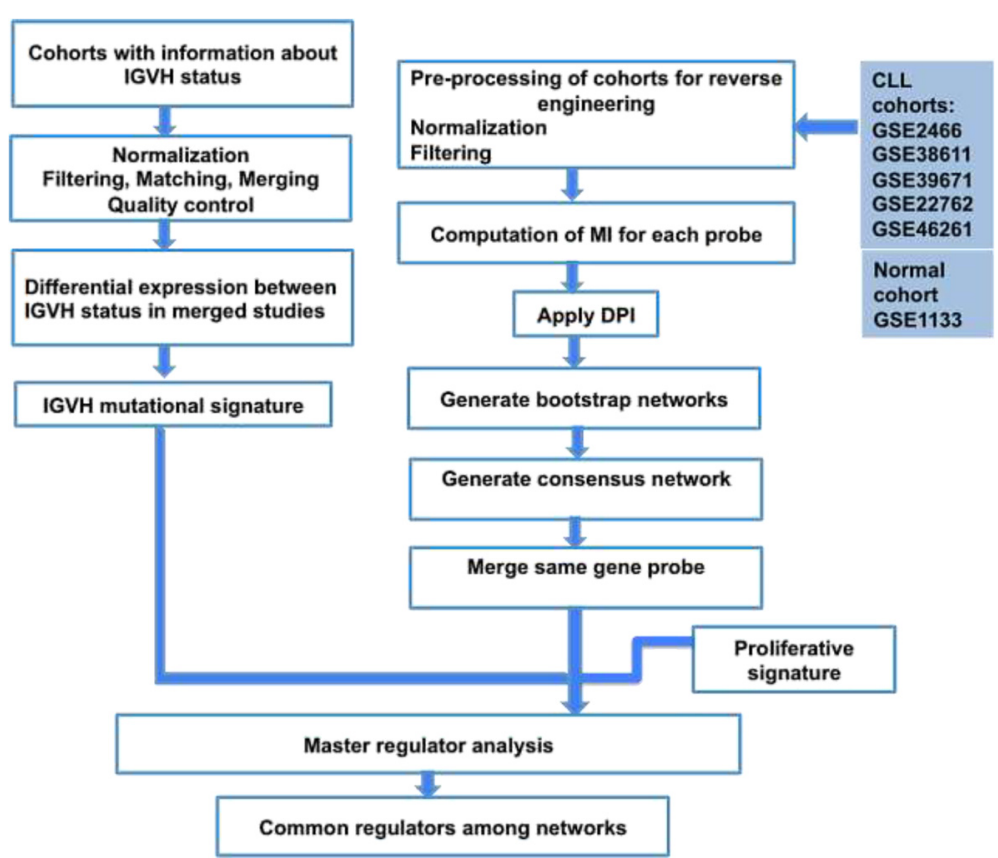

Fig. 1 The workflow used for reconstructing the CLL regulatory networks and for regulator inference 


\section{Dataset}

The present study used microarray gene expression data retrieved from the NCBI Gene Expression Omnibus (GEO) Database [20]. A total of 474 CLL patients from eleven studies were included in the differential expression analysis (Table 1). Each chosen dataset possessed available raw expression files (CEL files) and information regarding the subjects' IGVH mutational status. Five cohorts with 100 or more CLL patients were used to reverse engineer the transcriptional networks.

\section{Data preprocessing and IGVH mutational status signature}

Probe level normalization was performed independently in each cohort using the VSN method [21]. Quality checks were performed before and after the normalization process. To obtain a robust result, we applied a gene filtering procedure to each study level, which removed $30 \%$ of the non-expressed genes based on the mean intensity values and $30 \%$ of the non-informative genes with a small variation based on variance.

Usually, different microarray platforms have multiple probes (or probe sets) that represent the same gene transcript; therefore, gene matching is necessary. For probe summarization, we used the "IQR" method, in which we selected the probe ID with the largest interquartile range (IQR) of the expression values to represent the gene. The number of genes in each study may be different; thus, we performed gene merging to extract the common genes across multiple cohorts. Additionally, we included some genes that appeared in $80 \%$ of the studies and were missing in $20 \%$ of the studies. The MetaDE package in $\mathrm{R}$ was used for filtering, matching and merging procedures [22].
To detect the differential expression between the IGVH subtypes, we used a microarray meta-analysis approach. This methodological framework increases the reliability and generalizability of results [23]. We used the "MetaOmics" software suite, which contains three unified $\mathrm{R}$ packages: MetaQC, MetaDE and MetaPath [22]. The MetaQC package [24] was used for determining the meta-analysis inclusion/exclusion criteria. MetaDE was used to apply various state-of-the-art genomic meta-analysis methods to detect differentially expressed (DE) genes, including the Fisher [25], Stouffer, adaptively weighted statistic (AW) [26], maximum $P$-value (maxP), and the rth ordered $P$-value (rOP) methods [27]. The R statistical environment [28] was used to perform all statistical analyses.

\section{Functional enrichment}

The MetaPath package, which performs pathway metaanalysis [29], was used to detect enriched pathways. The Genecodis server [30-32] was used to perform modular enrichment analysis. The method obtains co-occurrence annotations in the KEGG and Panther databases, the $P$ values are calculated through hypergeometric analysis corrected by FDR method.

\section{Reverse engineering of the transcriptional networks}

After VSN normalization, each cohort was filtered based on its standard deviation distribution (sd). Probes with a sd below the shortest interval that contained half of the data in the distribution were discarded before the network reconstruction. ARACNE mutual information networks [14] were built based on five expression cohorts. Each network had 100 or more samples and was processed with different platforms (GSE2466, GSE38611,

Table 1 Cohorts used in this study

\begin{tabular}{llll}
\hline Author & Platform & Samples (mut/unmut) & Accession \\
\hline Mosca L et al. [58] & HG_U133A & $60(23 / 37)$ & GSE16746 \\
Fabris S et al. [59] & HG_U133A & $60(24 / 36)$ & GSE9992 \\
Del Guidice et al. [60] & HG_U133A & $20(16 / 4)$ & GSE15777 \\
Jao Baptista et al. [61] & HG_U133_Plus_2 & $24(10 / 14)$ & GSE33135 \\
Saiya-Cork K et al. [62] & HG_U133_Plus_2 & $19(4 / 15)$ & GSE26526 \\
Haslinger C et al. [63] & HG_U95A & $100(51 / 49)$ & GSE2466 \\
Seifert M et al. [64] & HuGene 1.0 ST & $9(4 / 5)$ & GSE36907 \\
& HG_U133_Plus_2 & $10(5 / 5)$ & GSE12734 \\
Stamatopoulos B et al. [53] & HG_U133_Plus_2 & $14(8 / 6)$ & GSE29605 \\
Mukherjee P et al. [65] & HG_U133_Plus_2 & $22(10 / 12)$ & GSE38611 \\
Fabris S et al. [66] & HuGene 1.0 ST & $136(76 / 60)$ & GSE46261 \\
Ronchetti D et al. [67] & HuGene 1.0 ST & $211(127 / 83)$ & GSE39671 \\
Chuang HY et al. [68] & HG_U133_Plus_2 & $130($ NA) & GSE22762 \\
Herold T et al. [49] & HG_U133_Plus_2 & 107 (NA) & GSE1133 \\
Su Al et al. [40] & HG_U133A & 126 (normal samples) & \\
\hline
\end{tabular}


GSE39671, GSE22762, and GSE46261). ARACNE was used to infer the targets of 807 TFs that were represented in the gene expression profile. The algorithm uses information-theoretic methods to analyze physical transcriptional interactions between the TFs and their targets. ARACNE uses expression data to compute pairwise mutual information (MI) and employs a computationally efficient Gaussian kernel estimator. First, it eliminates interactions that are below a minimum MI threshold, and then the Data Processing Inequality (DPI) theorem is used to eliminate interactions that are considered sampling errors. $\mathrm{R}$ scripts from the original protocol calculated the kernel width and MI threshold parameters. The $P$ value to determine the MI threshold and the DPI tolerance were set to 0.05 and $0 \%$, respectively. One hundred bootstrap datasets were used to create the bootstrap networks to accommodate the microarray data noise and the MI estimation error. A consensus network was then constructed by retaining edges that were supported across a significant number of the bootstrap networks. The entire process was also executed in normal tissues (GSE1133) as negative controls. The ARACNE algorithm was implemented in the Perl language by using the original procedure that was proposed by Margolin et al. [14], who also described the mathematical formulation of the algorithm.

\section{Regulator analysis}

Master regulator analysis (MRA) [33] of the reconstructed networks was used to identify (TFs) that regulate an IGVH mutational status signature. Enrichment was evaluated using the Fisher's exact test (FET); therefore, for each TF, the statistical significance of the intersection between the TF targets, which was represented in the ARACNE-generated network, and the list of differentially expressed genes was computed with the FET. These generated TFs were selected as candidate regulators of IGVH status. To avoid the possibility of finding non-specific CLL regulators and to exclude those that were directly involved with proliferation, we ran an MRA on both the CLL IGVH signature, to query a healthy tissue network, and a proliferative gene signature that was developed by Venet et al. [34], which is known as a meta-PCNA signature. To validate the power of the process for detecting significant regulons, we compared the common TFs among the reconstructed networks. MRA was performed using geWorkbench, a free, opensource genomic analysis platform [35].

\section{The clinical relevance and survival analyses}

We applied the Global Test [36] to determine the association between expression profiles and survival. For cases in which a significant association with patient outcome was observed, the gene list was reduced to derive a smaller prognostic signature with the intention of providing a profile with potential clinical use. The smaller signature was constructed using Cox proportional hazards models and clustering analysis as proposed by Goeman JJ and Finos L [37]. Briefly, the methodology is based on regression models in which the distribution of the response variable (overall survival or time to treatment) is modeled as a function of the covariates (expression values) [36]. The covariates were ordered in a hierarchical cluster with the absolute correlation distance and the method average linkage. To reduce the profile or "zoom" in on the significant results, the process discards non-significant branches from the dendrogram with the corresponding covariates. The MLInterfaces package [38] was used to construct an SVM (support vector machine) [39] to assess the predictive power of the reduced profile. We used a 218-sample training set that was chosen at random from patients with good and poor prognoses and a 218-sample test set to calculate the classification error.

Pairwise t-tests were applied with Bonferroni $P$ value corrections to compare the relative expression levels between two groups.

\section{Results}

\section{The IGVH mutational status expression profile}

Using a combination of CLL expression profiles, we used a microarray meta-analysis approach to obtain an IGVH mutational status signature. After preprocessing and quality control, we obtained 12,487 genes in 436 CLL patients from eight different cohorts, which included 218 unmuted and 218 mutated IGVH samples.

GSE26525 and GSE36907 were determined to be of lower quality after six quantitative quality control (QC) measures were taken into consideration; therefore, they were removed from the meta-analysis. The QC measures utilized were proposed by Kang et al. [24] and included: covering the internal homogeneity of coexpression structures among studies, the external consistency of coexpression patterns within a pathway database, and the accuracy and consistency of expressed gene detection or enriched pathway identification. Each QC index was used to identify low-quality studies and to determine whether they should be excluded from the meta-analysis.

The Fisher $P$-value method detected a significant number of genes and was used in the meta-analysis for differential expression. Table 2 shows the top 20 genes with differential expression between the IGVH statuses as determined using the Fisher method, and these are listed in order of statistical test and significance level. The total list of genes that were determined with statistically significant differences can be found in Additional file 1: Table S1. Figure 2 shows the expression distribution of the first 20 differentially expressed genes that were found with the meta-analysis with respect to IGVH 
Table 2 The top differentially expressed genes obtained with the meta-analysis

\begin{tabular}{llc}
\hline Gene & Regulation in poor prognoses & Corrected P-value \\
\hline CRY1 & Up & $4.78 \mathrm{e}-19$ \\
LPL & Up & $4.78 \mathrm{e}-19$ \\
ZBTB20 & Down & $4.78 \mathrm{e}-19$ \\
SEPT10 & Up & $4.78 \mathrm{e}-19$ \\
COBLL1 & Down & $4.78 \mathrm{e}-19$ \\
NRIP1 & Down & $4.78 \mathrm{e}-19$ \\
DMD & Up & $4.78 \mathrm{e}-19$ \\
ZAP70 & Up & $4.78 \mathrm{e}-19$ \\
LDOC1 & Up & $4.78 \mathrm{e}-19$ \\
WSB2 & Up & $4.78 \mathrm{e}-19$ \\
CLEC2B & Up & $4.78 \mathrm{e}-19$ \\
PCDH9 & Up & $4.78 \mathrm{e}-19$ \\
TCF7 & Down & $4.78 \mathrm{e}-19$ \\
PHEX & Up & $4.78 \mathrm{e}-19$ \\
SLAMF1 & Down & $4.78 \mathrm{e}-19$ \\
BCL7A & Up & $4.78 \mathrm{e}-19$ \\
PFKP & Up & $4.78 \mathrm{e}-19$ \\
ATOX1 & Up & $4.78 \mathrm{e}-19$ \\
USP6NL & Down & $4.78 \mathrm{e}-19$ \\
FUT8 & Down & $4.78 \mathrm{e}-19$ \\
SPG20 & Up & $4.78 \mathrm{e}-19$ \\
TGFBR3 & Up & $4.78 \mathrm{e}-19$ \\
CERS6 & Up & $4.78 \mathrm{e}-19$ \\
FLNB & Up & $4.78 \mathrm{e}-19$ \\
P2RX1 & Up & $4.78 \mathrm{e}-19$ \\
MYBL1 & Down & $4.78 \mathrm{e}-19$ \\
RNF41 & Up & $4.78 \mathrm{e}-19$ \\
\hline & Up & $4.78 \mathrm{e}-19$ \\
IFI4 & Up 19 \\
\hline
\end{tabular}

status. Significant differences between the sample groups were observed for all the top genes $(P>0.001$, two-tailed pairwise $t$-test with Bonferroni corrections).

Using a modular enrichment analysis with the KEGG and Panther pathways and the list of differentially expressed genes $(p<0.05)$, the following co-occurrence annotations were found with low significant $P$ values: pathways in cancer, focal adhesion, $\mathrm{T}$ cell receptor signaling, angiogenesis, and MAPK signaling. Pathway detection using the MetaPath package and collections from the molecular signatures database (MSigDB) showed a significant result for the GO terms that were related to extracellular signaling (q-value $>0.2$ ). The following terms were found to be statically significant: proteinaceous extracellular matrix, extracellular region, extracellular space, extracellular matrix, and extrinsic to membrane.

\section{Reconstruction of the CLL transcriptional networks and regulator analysis}

In Table 3, we list the datasets that were used for the regulatory network reconstructions, including the number of probes after filtering, which ensured the use of highly variable probes, and the large number of interactions (edges) and targets (nodes) found in each network after the reconstruction.

To improve the specificity of the regulator analysis, we conducted control processes. We used the IGVH status signature to query a healthy tissue network (GSE1133) [40] and used it as a control to detect regulators that were not tissue-specific. In addition, to exclude the regulators that were involved with proliferation, we performed the MRA within the CLL networks using as query a PCNA proliferative gene signature [34]. No inferred TF was enriched in the healthy control networks, nor were any TFs involved with the proliferative signature that was tested. Therefore, the specificity checks confirmed that the TFs involved in the prediction were related to CLL pathology.

The MRA identified a relatively small number of regulators in each network, and some variation was observed among them (Table 4). In total, 35 TFs were identified after taking into account all networks. With respect to the pathways enriched within this group of TFs, a cooccurrence annotation was found for Wnt signaling (0.00018). In spite of this variation, the following TFs emerged as regulators in at least four networks: CERS6 (80 targets), TCF7 (95 targets), and MYBL1 (59 targets). The number shown in parenthesis includes the targets in total for all the networks. Overlap among these selected regulators and their targets was found, in which 20 to 28 target genes were shared; consequently, multiple genes in the IGVH signature were co-regulated by several TFs (Additional file 1: Table S2).

\section{The clinical relevance of regulators with respect to survival}

Expression profiles were tested to evaluate their relationship with outcome using the GSE22762 $(n=107)$ and GSE39671 $(n=130)$ datasets. As seen in Table 4, some TF targets were found in common in the reconstructed networks. CERS6, TCF7 and MYBL1 stood out, suggesting a unifying process in CLL progression. Therefore, we focused on these regulons for interpretation. Every profile (TF targets) independently tested exhibited significance for survival with the following respective $P$ values for OS and TTT: TCF7 $(P=4.21 \mathrm{e}-08, P=$ 0.0046), CERS6 $(P=3.13 \mathrm{e}-06, P=0.015)$ and MYBL1 $(P=5.04 \mathrm{e}-5 ; P=0.002)$.

Then, we tested whether CERS6, TCF7, MYBL1 and their targets as a group, which included a total of 166 genes, could be related to patient outcomes. We found a 


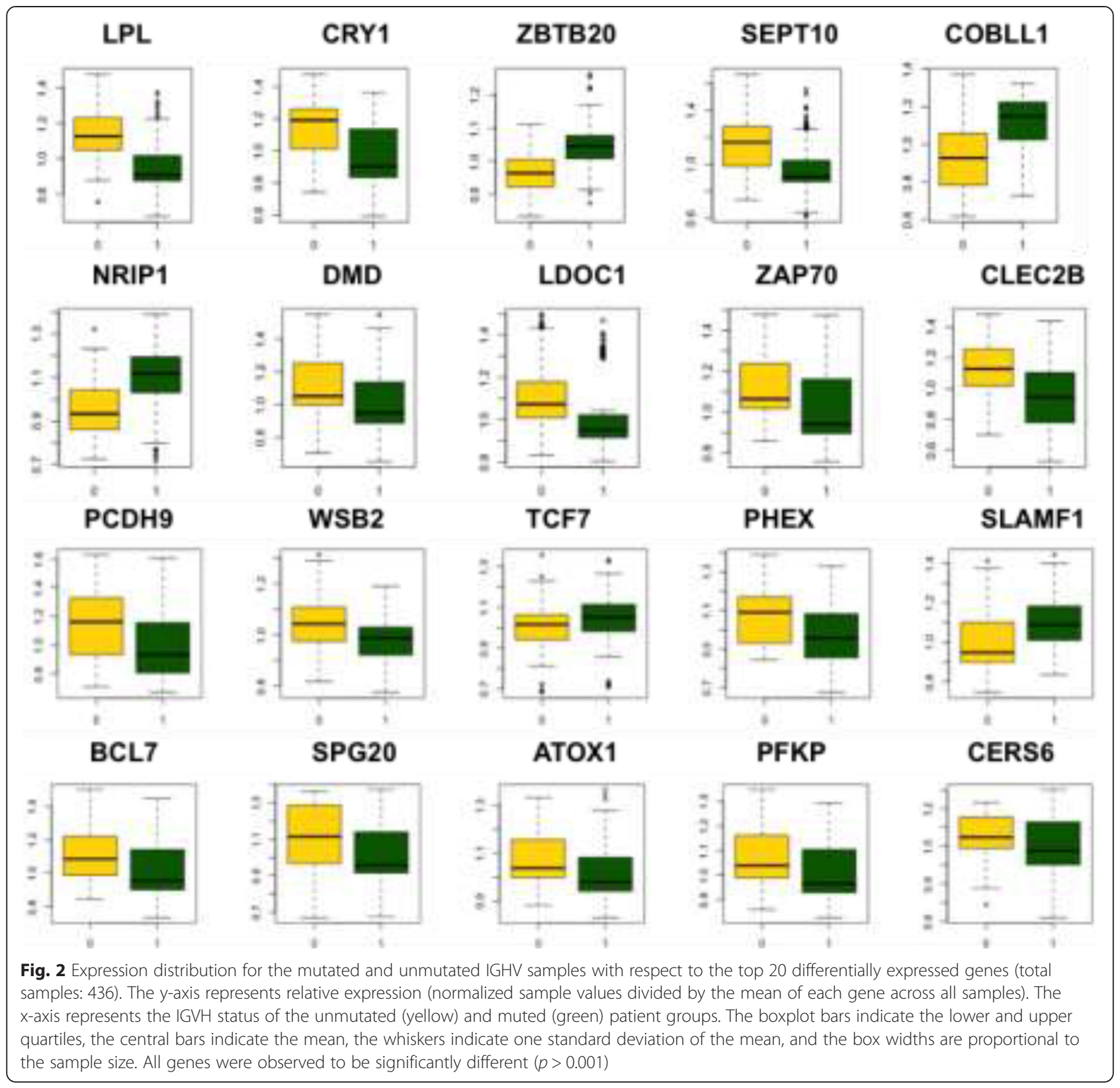

Table 3 Metrics of the reconstructed networks

\begin{tabular}{|c|c|c|c|c|c|}
\hline Dataset accession & No. of samples & Array platform & No. probes (original array /after filtering) & Nodes & Edges \\
\hline GSE2466 & 100 & HG_U95A & $12,626 / 8,879$ & 6,494 & 27,207 \\
\hline GSE38611 & 136 & HuGene 1.0 ST & $32,321 / 22,454$ & 17,271 & 110,952 \\
\hline GSE39671 & 130 & HG_U133_Plus_2 & $54,675 / 39,255$ & 12,115 & 78,351 \\
\hline GSE22762 & 107 & HG_U133_Plus_2 & $54,675 / 38,868$ & 16,471 & 169,016 \\
\hline GSE46261 & 211 & HuGene 1.0 ST & $32,321 / 12,968$ & 12,289 & 57,587 \\
\hline GSE1133 & 126 & HG_U133A & $22,283 / 13,941$ & 9,428 & 51,609 \\
\hline
\end{tabular}


Table 4 Transcription factors in CLL regulatory networks after MRA

\begin{tabular}{|c|c|c|c|c|c|c|c|}
\hline \multicolumn{4}{|l|}{ Network GSE46261 } & \multicolumn{4}{|l|}{ Network GSE39671 } \\
\hline Master regulator & FET $P$-value & Inter-section set & Mode & Master regulator & FET $P$-value & Inter-section set & Mode \\
\hline CERS6 & $9.01 \mathrm{E}-36$ & 40 & + & EGR3 & 7.36E-17 & 39 & - \\
\hline TCF7 & 1.17E-28 & 37 & - & CERS6 & $8.80 \mathrm{E}-17$ & 47 & + \\
\hline TLE1 & $1.66 \mathrm{E}-16$ & 22 & + & PHTF1 & $1.22 \mathrm{E}-11$ & 37 & + \\
\hline ZNF135 & 3.97E-15 & 21 & + & AEBP1 & 4.51E-10 & 30 & + \\
\hline MYBL1 & 4.33E-13 & 22 & - & NR2F6 & $4.58 \mathrm{E}-08$ & 20 & + \\
\hline ELK3 & $3.59 \mathrm{E}-10$ & 16 & + & MYBL1 & 7.17E-08 & 26 & - \\
\hline AEBP1 & $1.11 \mathrm{E}-09$ & 16 & + & ZNF91 & $8.43 \mathrm{E}-08$ & 16 & - \\
\hline GFI1 & 3.00E-09 & 13 & + & TFDP1 & $3.64 \mathrm{E}-07$ & 16 & + \\
\hline NCOR2 & 4.86E-08 & 12 & + & TCF7 & 5.57E-07 & 42 & - \\
\hline TRPS1 & $6.00 \mathrm{E}-08$ & 11 & + & APEX1 & $2.00 \mathrm{E}-06$ & 24 & + \\
\hline TSHZ2 & $3.55 \mathrm{E}-07$ & 16 & - & ZNF354A & $3.26 \mathrm{E}-06$ & 10 & + \\
\hline ARID5B & $6.01 \mathrm{E}-07$ & 9 & - & SMARCA4 & $6.54 \mathrm{E}-06$ & 39 & + \\
\hline NOD2 & 7.06E-07 & 17 & + & Network GSE38611 & & & \\
\hline EGR3 & $1.20 \mathrm{E}-06$ & 9 & - & MYBL1 & $3.80 \mathrm{E}-07$ & 6 & - \\
\hline ZNF236 & $2.13 \mathrm{E}-06$ & 8 & + & CERS6 & $5.90 \mathrm{E}-06$ & 4 & + \\
\hline HOXB2 & $2.76 \mathrm{E}-06$ & 9 & + & Network GSE22762 & & & \\
\hline BRIP1 & $3.27 \mathrm{E}-06$ & 15 & - & TCF7 & $3.01 \mathrm{E}-23$ & 49 & - \\
\hline MTA1 & $6.51 \mathrm{E}-06$ & 14 & + & EGR3 & $5.25 \mathrm{E}-14$ & 23 & - \\
\hline Network GSE2466 & & & & CERS6 & $1.21 \mathrm{E}-11$ & 21 & + \\
\hline KLF7 & $2.11 \mathrm{E}-13$ & 23 & - & MYBL1 & $3.38 \mathrm{E}-10$ & 24 & - \\
\hline AFF1 & $1.79 \mathrm{E}-08$ & 16 & - & TCF7L2 & $3.82 \mathrm{E}-10$ & 31 & - \\
\hline TCF3 & $1.26 \mathrm{E}-07$ & 21 & + & MYBL2 & 8.67E-10 & 23 & + \\
\hline TCF7 & 1.77E-05 & 18 & - & AEBP1 & $1.89 \mathrm{E}-09$ & 16 & + \\
\hline TLE1 & $2.72 \mathrm{E}-05$ & 8 & + & TFEC & $6.31 \mathrm{E}-08$ & 12 & - \\
\hline RUNX3 & $5.22 \mathrm{E}-05$ & 13 & - & PHTF1 & $8.18 \mathrm{E}-08$ & 29 & + \\
\hline ZNF135 & 1.10E-04 & 8 & + & PBX3 & 7.17E-07 & 14 & + \\
\hline KLF10 & $1.85 \mathrm{E}-04$ & 6 & + & EP400 & 7.72E-07 & 17 & + \\
\hline CERS6 & $1.85 \mathrm{E}-04$ & 6 & + & PPARD & 5.82E-06 & 25 & - \\
\hline
\end{tabular}

significant difference for OS $(P=2.46 \mathrm{e}-07)$ and for TTT $(P=0.00548)$. In Table 5 , genes associated with OS survival are listed in order of significance, as well as the direction of their regulation, and genes with statistical significance in both cohorts are underlined. TCF7 had prognostic value in both cohorts, and it was the secondmost statistically significant gene in the GSE22762 cohort. Additionally, NRIP1 and PDE8A were at the top of the list. All three genes were downregulated with respect to poor prognosis. Enrichment analysis using the 166 genes showed that focal adhesion $(2.3 \mathrm{e}-08)$ and $\mathrm{T}$ cell receptor signaling (2.6e-06) were the most implicated pathways, and the MAPK and Wnt signaling pathways, among others, were also detected with significant corrected $P$ values.

We next reduced the expression profile to develop a smaller prognostic gene signature using a Cox proportional hazards model. From the 166 genes, the procedure reduced the profile to 20 genes with positive or negative associations with survival. As shown in Fig. 3, the genes are ordered in a hierarchical clustering graph, which only shows the significant branches of the reduced profile. Notably, NRIP1 had the highest statistical significance, followed by TCF7, and the high expression of both genes was associated with survival. In other words, low expression was associated with a poor prognosis.

To determine whether the CERS6, TCF7 and MYBL1 expression levels were related to IGVH mutational status, we used pairwise t-tests to analyze the complete dataset that was previously used for the meta-analysis. We found that TCF7 and MYBL1 expression was significantly lower in the unmutated IGVH status patients than in the mutated IGVH status patients. Moreover, CERS6 expression was higher in the unmutated patients, 
Table 5 Transcription factors and targets with significant overall survival associations in cohort GSE22762

\begin{tabular}{|c|c|c|c|c|c|}
\hline GENE & Regulation in poor prognoses & $P$-value & GENE & Regulation in poor prognoses & $P$-value \\
\hline NRIP1 & Down & $1.89 \mathrm{E}-10$ & $\mathrm{BCL} 7 \mathrm{~A}$ & Up & $9.31 \mathrm{E}-04$ \\
\hline$\underline{\text { TCF7 }}$ & Down & $1.67 \mathrm{E}-06$ & SNED1 & Down & 1.09E-03 \\
\hline PDE8A & Down & 7.10E-06 & DOK2 & Down & 1.10E-03 \\
\hline CD247 & Down & 7.67E-06 & ARSD & Up & $1.14 \mathrm{E}-03$ \\
\hline$\underline{\text { SORL1 }}$ & Down & 4.27E-05 & $\underline{\text { RASGRP1 }}$ & Down & 1.27E-03 \\
\hline$\underline{\text { ATOX1 }}$ & Up & 5.07E-05 & LHFPL2 & Down & $1.54 \mathrm{E}-03$ \\
\hline $\mathrm{P} 2 \mathrm{RX} 1$ & Up & $6.37 \mathrm{E}-05$ & EGR3 & Down & $1.65 \mathrm{E}-03$ \\
\hline NME1 & Up & $8.27 \mathrm{E}-05$ & HLADMA & Up & $1.85 \mathrm{E}-03$ \\
\hline NMB & Up & $1.12 \mathrm{E}-04$ & LRMP & Up & $1.96 \mathrm{E}-03$ \\
\hline GMDS & Up & $1.17 \mathrm{E}-04$ & $\mathrm{DIP} 2 \mathrm{C}$ & Up & 2.06E-03 \\
\hline IL2RB & Down & $1.34 \mathrm{E}-04$ & TMED3 & Up & $3.24 \mathrm{E}-03$ \\
\hline AAK1 & Down & $1.61 \mathrm{E}-04$ & $\underline{\text { MYBL1 }}$ & Down & $3.29 \mathrm{E}-03$ \\
\hline ME2 & Up & $1.76 \mathrm{E}-04$ & PHEX & Up & $3.81 \mathrm{E}-03$ \\
\hline SERPINF1 & Up & $3.00 \mathrm{E}-04$ & UGT8 & Up & 4.98E-03 \\
\hline FARP1 & Up & $3.27 \mathrm{E}-04$ & $\underline{\mathrm{SDC} 3}$ & Down & $5.25 \mathrm{E}-03$ \\
\hline NUCB2 & Down & $3.58 \mathrm{E}-04$ & SFTPB & Up & $6.06 \mathrm{E}-03$ \\
\hline HOMER2 & Up & $5.74 \mathrm{E}-04$ & PEBP1 & Up & $6.34 \mathrm{E}-03$ \\
\hline$\underline{\mathrm{SLC} 16 \mathrm{A6}}$ & Down & $5.75 E-04$ & $\underline{\mathrm{LPL}}$ & Up & $6.98 \mathrm{E}-03$ \\
\hline SYNJ2 & Down & $9.05 \mathrm{E}-04$ & LDOC1 & Up & 7.04E-03 \\
\hline SLAMF1 & Down & $9.20 \mathrm{E}-04$ & & & \\
\hline
\end{tabular}

Genes with significant time to treatment associations in cohort GSE39671 are underlined

indicating that these genes play an important role in disease prognosis (Fig. 4).

An SVM classifier was constructed for the common regulators (TCF7, CERS6, and MYBL1) and their targets, and the top 50 IGVH signature DE genes. We compared the dataset previously used in the meta-analysis (436 samples) with the classification error rates from the confusion matrix. The regulators and the 50-gene signature performed similarly, although a slightly lower error was observed in the signature $(0.09174312$ vs. 0.05504587$)$. These classification errors indicate that the regulators and their targets exhibited prognostic utility.

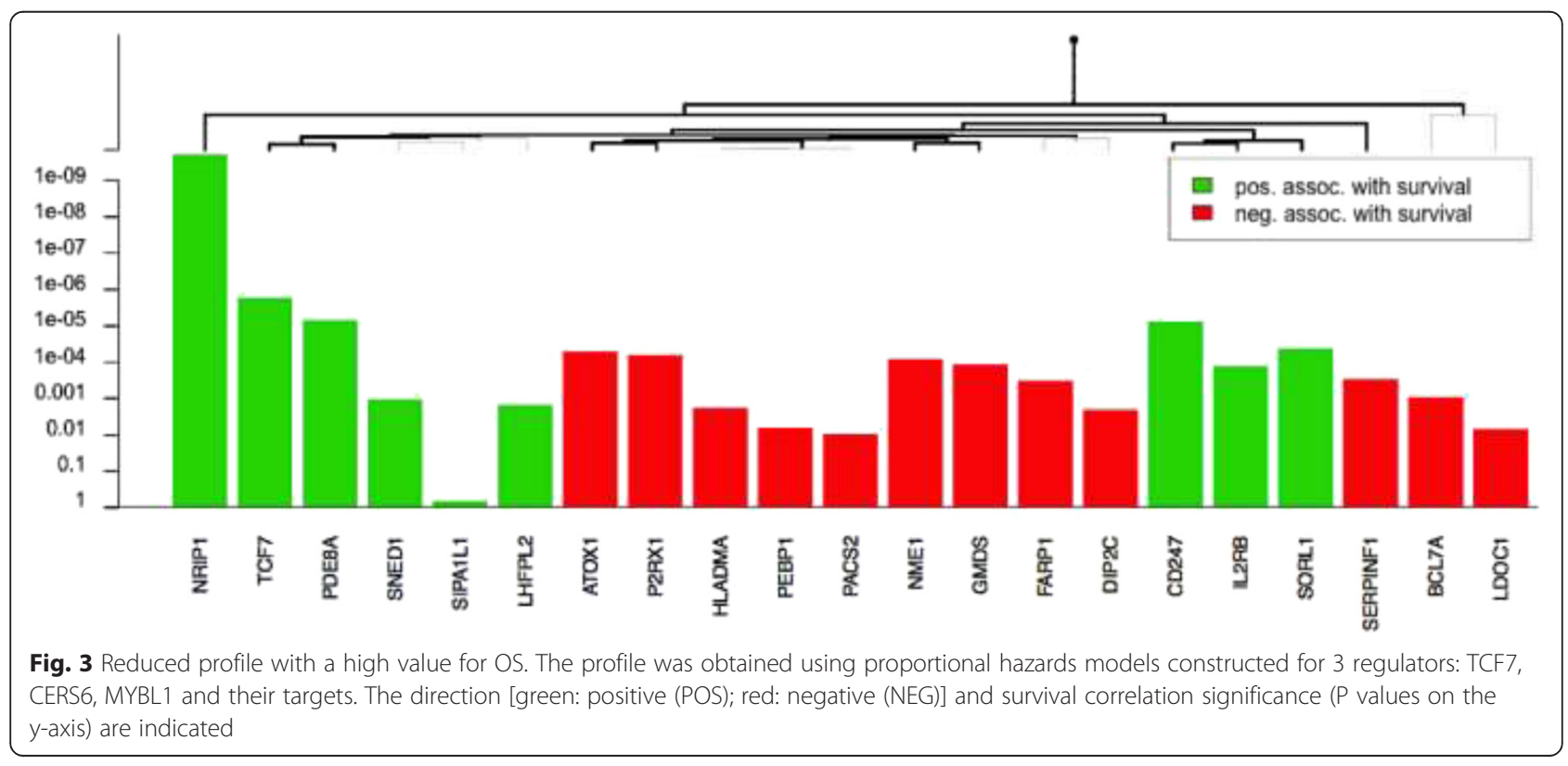



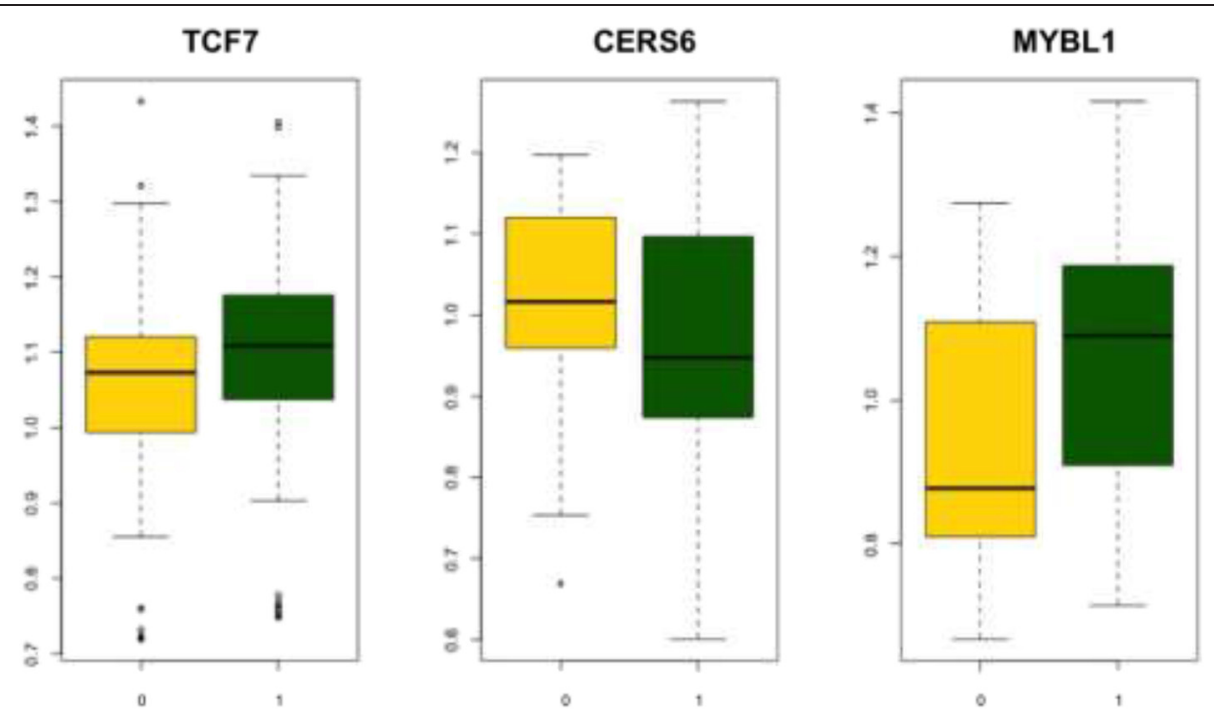

Fig. 4 TCF7, CERS6, and MYBL1 expression levels from the integrated dataset (total samples: 436). The y-axis represents relative expression (normalized sample values divided by the mean of each gene across all samples). The $x$-axis represents the unmutated (yellow) and muted (green) IGVH statuses. The boxplot bars indicate the lower and upper quartiles, the central bars indicate the mean, the whiskers indicate one standard deviation from the mean, and the box widths are proportional to the sample sizes. All genes were significantly different ( $p>0.001$, two-tailed pairwise t-tests with Bonferroni corrections) between the sample groups

\section{Discussion}

To identify prognostic markers in CLL, a robust IGVH mutational signature was generated and used to infer its upstream TFs through mutual information networks and master regulator analysis. Given the large number of samples used and the heterogeneous spectrum of genomic aberrations represented, the integrated information allowed us to bring together various molecular events that underlie CLL and to compare patients only by their IGVH mutational status.

Because the IGVH mutational status is a good survival predictor, genes that are differentially expressed in mutated versus unmutated subtypes are also meaningful in prognosis. The top differentially expressed genes found in this study have been previously associated with IGVH status [7-9]. Genes such as LPL, CRY1, ZBTB20, SEPT10, COBLL1, NRIP1, DMD, LDOC1, and ZAP70 were found in the top DE list and have been shown to have CLL prognostic value [41, 42].

The differentially expressed genes between the IGVH subtypes were preferentially enriched with the pathways that are related to extracellular signaling. As was shown in the pathway meta-analysis, no specific differences between the subtypes at the specific pathway level were observed; therefore, it is possible that a significant overlap in the molecular characteristics in the IGVH subtypes were present. Previous expression profiling that was conducted with small patient numbers showed a common gene expression signature and reduced number of differentially expressed genes between IGVH subtypes $[7,8]$. Although the number of differentially expressed genes increased in our meta-analysis approach, no difference was observed in the particular pathways beyond the terms related with extracellular signaling. These findings suggest that both subtypes of the disease are derived from a common origin or common transformation mechanism. It has previously been suggested that the IGVH subtypes derive from progenitors that are reminiscent of antigen-experienced B cells given the similar expression profiles [7], a model supported by frequent B cell receptor repertoire skewing and stereotypy [43].

The modular enrichment analysis executed directly with all the differentially expressed genes, improved the resolution of the pathway analysis, co-occurrence annotations were found with low significant $P$ values for: pathways in cancer, focal adhesion, $\mathrm{T}$ cell receptor signaling, angiogenesis, and MAPK signaling. The pathways involved suggest the cellular origin of CLL. Encounter of naive $B$ cells with antigen may progress either through a $\mathrm{T}$ cell-dependent reaction or in $\mathrm{T}$ cell-independent immune response. Possibly the mutated IGVH subtype is derived from the post-germinal center, generating memory $\mathrm{B}$ cells that have undergone somatic hypermutation of IGHV genes, unlike the unmutated subtype, which has not passed through the germinal center reaction, leading to the formation of antigen-experienced $\mathrm{B}$ cells harboring unmutated IGHV genes [2]. The expansion of CLL cells may be due to the accumulation of genetic lesions that confer higher aggressiveness, as well as interactions with the micro-environmental and antigens through the $\mathrm{BCR}$, that promote signaling associated with cell proliferation and apoptosis inhibition [43]. 
Some differences in the chromosomal rearrangements between the subtypes, such as $17 \mathrm{q}$ and $11 \mathrm{q}$ deletions, as well as mutations in the ATM, are thought to be associated with the unmutated subtype, while less severe changes, such as $13 \mathrm{q}$ deletions, are associated with the mutated IGVH subtype. Additionally, the use of certain $\mathrm{VH}$ genes suggests differences in antigenic stimulation (i.e., VH 1-69 in the non-mutated subtype vs. VH 3-7, 4-34 in the mutated subtype) [44]. Nonetheless, it is not clear if the differences mentioned above are caused by the mutational IGVH status or are associated with it.

CERS6, TCF7, and MYBL1 stood out as common regulators in at least four networks, all three TFs have been implicated in the cancer process. CERS6 is involved in pro-apoptotic responses [45], epithelial-tomesenchymal transition, plasma membrane fluidity and cell motility [46].

TCF7 is a member of a family of HMG-box-containing factors that are known to associate with $\beta$-catenin in the nucleus to mediate Wnt signaling. The Wnt signaling pathway is activated in CLL, and our data strengthens its role in prognosis. Uncontrolled Wnt signaling may contribute to the defective apoptosis that characterizes this malignancy [47]. Recent evidence regarding the role of TCF7 in CLL has been reported; in multivariate analyses of CLL patients, Kienle et al. [48] found evidence for the role of TCF7 in genetic risk defined by IGHV status, V3-21 usage, 11q-, 17p- and survival. Herold et al. [49] proposed an eight-gene prognostic score for CLL that included TCF7, NRIP1, and PDE8A for the prediction of survival and TTT. Here, we observed that these three genes were highly associated with survival using a completely different methodological approach and the same cohort (GSE22762). Conversely, Bou et al. [50] proposed a risk score combining with NRIP1 and TCF7 expression to identify high-risk patients. Therefore, the involvement of these genes in disease prognosis is significant.

The TCF7 targets inferred in this work were compared with the results of $\mathrm{Wu}$ et al. [51], who found that this TF was implicated in self-renewal and differentiation switch in early hematopoietic precursors. The authors used ChIP-Seq analysis to identify target genes bound by TCF7 in a multipotential hematopoietic cell line. Thirtynine inferred targets in our work were identified by ChIP-Seq analysis in the above-mentioned work. Of these, the following are part of the reduced profile: BCL7A, CD247, GMDS, LHFPL2, NME1, NRIP1, and TCF7. To some extent, these results validate the inferred interactions.

Another regulator that was consistently found in several networks was MYBL1. This is a TF that plays a role in B-cell hematological malignancies [52]. Stamatopoulos et al. [53] found that MYBL1 expression predicted overall survival in CLL patients in the context of ZAP70 expression. As evident in the IGVH signature developed here (Table 2), MYBL1 was under-expressed in the unmutated subtype, emerged as a key regulator in the process of regulator inference (Table 4), and was associated with poor prognosis (Table 5). Interestingly, MYBL1 is specifically expressed by centroblasts [54]; therefore, this gene may be involved in the centro-germinal reaction and may support the cellular origin of CLL. On the other hand, common mechanisms should operate in CLL and diffuse large B-cell lymphoma (DLBCL) because, for example, some cases of DLBCL occur in a CLL background (Richter's Syndrome). Interestingly, MYBL1 is part of a gene expression-based risk score in DLBCL [55], and it is included in outstanding molecular signatures developed for molecular subclassification in DLBCL [56, 57].

It is recognized that the overlap between the expression studies, particularly with respect to the prognostic signatures, is not perfect and contains only a few genes. Prognostic signatures have proven to be study dependent and inconsistent. Lim WK et al. [11] argued that genes in prognostic signatures act as passengers rather than drivers of the phenotypic differences. The genes that are most differentially expressed among the phenotypic states tend to be downstream from the determinants of the differences. Due to the complex interplay of regulatory interactions, these downstream genes are unstable. Therefore, it is of interest to search for transcription factors that regulate prognostic signatures.

\section{Conclusions}

Regulatory network reconstructions allowed us to identify candidate regulators for an IGVH signature and uncover markers with prognostic implications. Moreover, with the nature of the methodological process, our results provide some insight into the regulatory programs that are involved.

\section{Additional file}

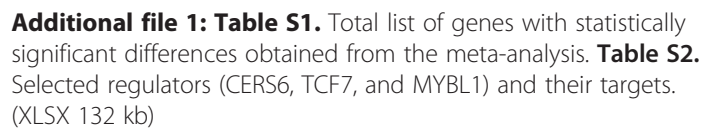

\section{Abbreviations}

CLL: Chronic lymphocytic leukemia; IGHV: Immunoglobulin variable region heavy chain; DE: Differentially expressed; TF: Transcription factor; MRA: Master regulator analysis; SVM: Support vector machine; OS: Overall survival; TाT: Time to treatment.

\section{Competing interests}

The authors declare that they have no competing interests. 


\section{Authors' contributions}

SY conceived and designed the study, performed analyses, interpreted the data and wrote the manuscript. MT coordinated the work and interpreted the data. $L L$ analyzed and reviewed the statistical and computational analyses. All authors read and approved the final manuscript.

\section{Acknowledgments}

Vicerectoria de Investigaciones and Facultad de Ciencias, Universidad de los Andes, supported this work.

\section{Author details}

${ }^{1}$ Facultad de Ciencias, Departamento de Ciencias Biológicas, Universidad de los Andes, Bogotá D.C., Colombia. ²Departamento de Estadística, Universidad Nacional de Colombia, Bogotá D.C., Colombia.

\section{Received: 9 February 2015 Accepted: 3 November 2015}

\section{Published online: 25 November 2015}

\section{References}

1. Zenz T, Mertens D, Küppers R, Döhner H, Stilgenbauer S. From pathogenesis to treatment of chronic lymphocytic leukaemia. Nat Rev Cancer. 2010;10:37-50.

2. Damle RN, Wasil T, Fais F, Ghiotto F, Valetto A, Allen SL, et al. Ig V gene mutation status and CD38 expression as novel prognostic indicators in chronic lymphocytic leukemia. Blood. 1999;94(6):1840-7.

3. Hamblin TJ, Davis Z, Gardiner A, Oscier DG, Stevenson FK. Unmutated Ig $\mathrm{V}(\mathrm{H})$ genes are associated with a more aggressive form of chronic lymphocytic leukemia. Blood. 1999;94(6):1848-54

4. Döhner $H$, Stielgenbauer $S$, Benner $A$, Leupolt $E$, Kröber $A$, Bullinger $L$, et al. Genomic aberrations and survival in chronic lymphocytic leukemia. N Engl J Med. 2000;343(26):1910-6.

5. Wang L, Lawrence MS, Wan Y, Stojanov P, Sougnez C, Stevenson K, et al. SF3B1 and other novel cancer genes in chronic lymphocytic leukemia. N Engl J Med. 2011;365(26):2497-506.

6. Wiestner A, Rosenwald A, Barry TS, Wright G, Davis RE, Henrickson SE, et al. ZAP-70 expression identifies a chronic lymphocytic leukemia subtype with unmutated immunoglobulin genes, inferior clinical outcome, and distinct gene expression profile. Blood. 2003;101(12):4944-51.

7. Klein U, Tu Y, Stolovitzky GA, Mattioli M, Cattoretti G, Husson H, et al. Gene expression profiling of B cell chronic lymphocytic leukemia reveals a homogeneous phenotype related to memory B cells. J Exp Med. 2001:194(11):1625-38.

8. Rosenwald A, Alizadeh AA, Widhopf G, Simon R, Davis RE, Yu X, et al. Relation of gene expression phenotype to immunoglobulin mutation genotype in B cell chronic lymphocytic leukemia. J Exp Med. 2001;194(11):1639-47.

9. Abruzzo LV, Barron LL, Anderson K, Newman RJ, Wierda WG, O'brien S, et al. Identification and validation of biomarkers of $\lg \mathrm{V}(\mathrm{H})$ mutation status in chronic lymphocytic leukemia using microfluidics quantitative real-time polymerase chain reaction technology. J Mol Diagn. 2007;9(4):546-55.

10. Bae T, Rho K, Choi JW, Horimoto K, Kim W, Kim S. Identification of upstream regulators for prognostic expression signature genes in colorectal cancer. BMC Syst Biol. 2013;7:86.

11. Lim WK, Lyashenko E, Califano A. Master regulators used as breast cancer metastasis classifier. Pac Symp Biocomput. 2009:504-15

12. Ein-Dor L, Kela I, Getz G, Givol D, Domany E. Outcome signature genes in breast cancer: is there a unique set? Bioinformatics. 2005:21(2):171-8.

13. Rhodes DR, Chinnaiyan AM. Integrative analysis of the cancer transcriptome. Nat Genet. 2005:37(Suppl):S31-7.

14. Margolin AA, Wang K, Lim WK, Kustagi M, Nemenman I, Califano A. Reverse engineering cellular networks. Nat Protoc. 2006;1(2):662-71.

15. Basso K, Margolin AA, Stolovitzky G, Klein U, Dalla-Favera R, Califano A, et al. Reverse engineering of regulatory networks in human B cells. Nat Genet. 2005:37(4):382-90

16. Basso K, Saito M, Sumazin P, Margolin AA, Wang K, Lim WK, et al. Integrated biochemical and computational approach identifies BCL6 direct target genes controlling multiple pathways in normal germinal center B cells. Blood. 2010;115(5):975-84.

17. Fletcher MN, Castro MA, Wang X, de Santiago I, O'Reilly M, Chin SF, et al. Master regulators of FGFR2 signalling and breast cancer risk. Nat Commun. 2013;4:2464.
18. Albanus RD, Juliani Siqueira Dalmolin R, Alves Castro MA, Augusto de Bittencourt Pasquali M, de Miranda Ramos V, Pens G, et al. Reverse engineering the neuroblastoma regulatory network uncovers MAX as one of the master regulators of tumor progression. PLoS ONE. 2013;8(12):e82457.

19. Agnelli L, Forcato M, Ferrari F, Tuana G, Todoerti K, Walker BA. The reconstruction of transcriptional networks reveals critical genes with implications for clinical outcome of multiple myeloma. Clin Cancer Res. 2011:17(23):7402-12.

20. GEO DataSets [http://www.ncbi.nlm.nih.gov/geo/ Accessed 1 Jun 2014.]

21. Huber W, von Heydebreck A, Sultmann H, Poustka A, Vingron M. Variance stabilization applied to microarray data calibration and to the quantification of differential expression. Bioinformatics. 2002;18 Suppl 1:S96-104.

22. Wang X, Kang DD, Shen K, Song C, Lu S, Chang LC, et al. An R package suite for microarray meta-analysis in quality control, differentially expressed gene analysis and pathway enrichment detection. Bioinformatics. 2012;28(19):2534-6.

23. Taminau J, Lazar C, Meganck S, Nowé A. Comparison of merging and metaanalysis as alternative approaches for integrative gene expression analysis. ISRN Bioinform. 2014;2014:345106.

24. Kang DD, Sibille E, Kaminski N, Tseng GC. MetaQC: objective quality contro and inclusion/exclusion criteria for genomic meta-analysis. Nucleic Acids Res. 2012:40(2):e15.

25. Rhodes DR, Barrette TR, Rubin MA, Ghosh D, Chinnaiyan AM. Metaanalysis of microarrays: interstudy validation of gene expression profiles reveals pathway dysregulation in prostate cancer. Cancer Res. 2002;62(15):4427-33.

26. Li J, Tseng GC. An adaptively weighted statistic for detecting differential gene expression when combining multiple transcriptomic studies. Ann Appl Stat. 2011:5:994-1019.

27. Song C, Tseng GC. Hypothesis setting and order statistic for robust genomic meta-analysis. Ann Appl Stat. 2014;8(2):777-800.

28. R Core Team. R: a language and environment for statistical computing. Vienna: R Foundation for Statistical Computing; 2013. ISBN 3-900051-07-0, http://www.R-project.org/. Accessed 1 Jun 2014.

29. Shen K, Tseng GC. Meta-analysis for pathway enrichment analysis when combining multiple genomic studies. Bioinformatics. 2010;26(10):1316-23.

30. Tabas-Madrid D, Nogales-Cadenas R, Pascual-Montano A. GeneCodis3: a non-redundant and modular enrichment analysis tool for functional genomics. Nucleic Acids Res. 2012;40(Web Server issue):W478-83.

31. Nogales-Cadenas R, Carmona-Saez P, Vazquez M, Vicente C, Yang X, Tirado $F$, et al. GeneCodis: interpreting gene lists through enrichment analysis and integration of diverse biologicalinformation. Nucleic Acids Res. 2009;37(Web Server issue):W317-22

32. Carmona-Saez P, Chagoyen M, Tirado F, Carazo JM, Pascual-Montano A. GENECODIS: a web-based tool for finding significant concurrent annotations in gene lists. Genome Biol. 2007:8:R3.

33. Lefebvre C, Rajbhandari P, Alvarez MJ, Bandaru P, Lim WK, Sato M, et al. A human B-cell interactome identifies MYB and FOXM1 as master regulators of proliferation in germinal centers. Mol Syst Biol. 2010;6:377.

34. Venet D, Dumont JE, Detours V. Most random gene expression signatures are significantly associated with breast cancer outcome. PLoS Comput Biol. 2011;7(10):e1002240.

35. Floratos A, Smith K, Ji Z, Watkinson J, Califano A. geWorkbench: an open source platform for integrative genomics. Bioinformatics. 2010;26(14):1779-80.

36. Goeman JJ, van de Geer SA, de Kort F, van Houwelingen HC. A global test for groups of genes: testing association with a clinical outcome. Bioinformatics. 2004;20(1):93-9.

37. Goeman JJ, Finos L. The inheritance procedure: multiple testing of treestructured hypotheses. Stat Appl Genet Mol Biol. 2012;11(1):Article 11.

38. Carey V, Gentleman R, Mar J, Vertrees cfJ, Gatto L. MLInterfaces: Uniform interfaces to $\mathrm{R}$ machine learning procedures for data in Bioconductor containers. R package version 1.46.0. https://www.bioconductor.org/ packages/release/bioc/html/MLInterfaces.html.

39. Schölkopf B, Smola AJ. Learning with kernels: support vector machines, regularization, optimization, and beyond. Cambridge: The MIT Press; 2002.

40. Su Al, Wiltshire T, Batalov S, Lapp H, Ching KA, Block D, et al. A gene atlas of the mouse and human protein-encoding transcriptomes. Proc Natl Acad Sci U S A. 2004;101(16):6062-7.

41. Oppezzo P, Vasconcelos $Y$, Settegrana C, Jeannel D, Vuillier F, LegarffTavernier M, et al. The LPL/ADAM29 expression ratio is a novel prognosis indicator in chronic lymphocytic leukemia. Blood. 2005;106(2):650-7 
42. van't Veer MB, Brooijmans AM, Langerak AW, Verhaaf B, Goudswaard CS, Graveland WJ, et al. The predictive value of lipoprotein lipase for survival in chronic lymphocytic leukemia. Haematologica. 2006;91(1):56-63.

43. Gaidano G, Foà R, Dalla-Favera R. Molecular pathogenesis of chronic lymphocytic leukemia. J Clin Invest. 2012;122(10):3432-8.

44. Fais F, Ghiotto F, Hashimoto S, Sellars B, Valetto A, Allen SL, et al. Chronic lymphocytic leukemia $B$ cells express restricted sets of mutated and unmutated antigen receptors. J Clin Invest. 1998;102(8):1515-25.

45. Schüll S, Günther SD, Brodesser S, Seeger JM, Tosetti B, Wiegmann K, et al. Cytochrome c oxidase deficiency accelerates mitochondrial apoptosis by activating ceramide synthase 6. Cell Death Dis. 2015;6:e1691.

46. Edmond V, Dufour F, Poiroux G, Shoji K, Malleter M, Fouqué A, et al. Downregulation of ceramide synthase- 6 during epithelial-to-mesenchymal transition reduces plasma membrane fluidity and cancer cell motility. Oncogene. 2015;34(8):996-1005.

47. Lu D, Zhao Y, Tawatao R, Cottam HB, Sen M, Leoni LM, et al. Activation of the Wnt signaling pathway in chronic lymphocytic leukemia. Proc Natl Acad Sci U S A. 2004;101(9):3118-23.

48. Kienle D, Benner A, Läufle C, Winkler D, Schneider C, Bühler A, et al. Gene expression factors as predictors of genetic risk and survival in chronic lymphocytic leukemia. Haematologica. 2010;95(1):102-9.

49. Herold T, Jurinovic V, Metzeler KH, Boulesteix AL, Bergmann M, Seiler T, et al. An eight-gene expression signature for the prediction of survival and time to treatment in chronic lymphocytic leukemia. Leukemia. 2011;25(10):1639-45.

50. Bou Samra E, Klein B, Commes T, Moreaux J. Identification of a 20-gene expression-based risk score as a predictor of clinical outcome in chronic lymphocytic leukemia patients. Biomed Res Int. 2014;2014:423174.

51. Wu JQ, Seay M, Schulz VP, Hariharan M, Tuck D, Lian J, et al. Tcf7 is an important regulator of the switch of self-renewal and differentiation in a multipotential hematopoietic cell line. PLoS Genet. 2012;8(3):e1002565.

52. Golay J, Facchinetti $V$, Ying G, Introna M. The A-myb transcription factor in neoplastic and normal B cells. Leuk Lymphoma. 1997;26(3-4):271-9.

53. Stamatopoulos B, Haibe-Kains B, Equeter C, Meuleman N, Sorée A, De Bruyn C, et al. Gene expression profiling reveals differences in microenvironment interaction between patients with chronic lymphocytic leukemia expressing high versus low ZAP70 mRNA. Haematologica. 2009;94(6):790-9.

54. Golay J, Broccoli V, Lamorte G, Bifulco C, Parravicini C, Pizzey A, et al. The A-Myb transcription factor is a marker of centroblasts in vivo. J Immunol. 1998;160(6):2786-93.

55. Bret $C$, Klein B, Moreaux J. Gene expression-based risk score in diffuse large B-cell lymphoma. Oncotarget. 2012;3(12):1700-10.

56. Alizadeh AA, Eisen MB, Davis RE, Ma C, Lossos IS, Rosenwald A, et al. Distinct types of diffuse large B-cell lymphoma identified by gene expression profiling. Nature. 2000;403(6769):503-11.

57. Lenz G, Wright G, Dave SS, Xiao W, Powell J, Zhao H, et al. Stromal gene signatures in large-B-cell lymphomas. N Engl J Med. 2008;359(22):2313-23.

58. Mosca L, Fabris S, Lionetti M, Todoerti K, Agnelli L, Morabito F, et al. Integrative genomics analyses reveal molecularly distinct subgroups of B-cell chronic lymphocytic leukemia patients with 13q14 deletion. Clin Cancer Res. 2010;16:5641-53.

59. Fabris S, Mosca L, Todoerti K, Cutrona G, Lionetti M, Intini D, et al. Molecular and transcriptional characterization of $17 p$ loss in B-cell chronic lymphocytic leukemia. Genes Chromosomes Cancer. 2008;47(9):781-93.

60. Del Giudice I, Chiaretti S, Tavolaro S, De Propris MS, Maggio R, Mancini F, et al. Spontaneous regression of chronic lymphocytic leukemia: clinical and biologic features of 9 cases. Blood. 2009;114(3):638-46.

61. Baptista MJ, Muntañola A, Calpe E, Abrisqueta P, Salamero O, Fernández E, et al. Differential gene expression profile associated to apoptosis induced by dexamethasone in CLL cells according to IGHV/ZAP-70 status. Clin Cancer Res. 2012;18(21):5924-33.

62. Saiya-Cork K, Collins R, Parkin B, Ouillette P, Kuizon E, Kujawski L, et al. A pathobiological role of the insulin receptor in chronic lymphocytic leukemia. Clin Cancer Res. 2011;17(9):2679-92.

63. Haslinger C, Schweifer N, Stilgenbauer S, Döhner H, Lichter P, Kraut N, et al Microarray gene expression profiling of B-cell chronic lymphocytic leukemia subgroups defined by genomic aberrations and VH mutation status. J Clin Oncol. 2004;22(19):3937-49.

64. Seifert M, Sellmann L, Bloehdorn J, Wein F, Stilgenbauer S, Dürig J, et al. Cellular origin and pathophysiology of chronic lymphocytic leukemia. J Exp Med. 2012:209(12):2183-98.
65. GEO DataSets [http://www.ncbi.nlm.nih.gov/geo/query/acc.cgi?acc=GSE29605]. Accessed 1 Jun 2014

66. Fabris S, Mosca L, Cutrona G, Lionetti M, Agnelli L, Ciceri G, et al. Chromosome $2 p$ gain in monoclonal B-cell lymphocytosis and in early stage chronic lymphocytic leukemia. Am J Hematol. 2013;88(1):24-31.

67. Ronchetti D, Mosca L, Cutrona G, Tuana G, Gentile M, Fabris S, et al. A Small nucleolar RNAs as new biomarkers in chronic lymphocytic leukemia. BMC Med Genomics. 2013;6:27.

68. Chuang HY, Rassenti L, Salcedo M, Licon K, Kohlmann A, Haferlach T, et al. Subnetwork-based analysis of chronic lymphocytic leukemia identifies pathways that associate with disease progression. Blood. 2012;120(13):2639-49.

\section{Submit your next manuscript to BioMed Central and take full advantage of:}

- Convenient online submission

- Thorough peer review

- No space constraints or color figure charges

- Immediate publication on acceptance

- Inclusion in PubMed, CAS, Scopus and Google Scholar

- Research which is freely available for redistribution 